Proyecciones

Vol. 19, No 3, pp. 207-226, December 2000.

Universidad Católica del Norte

Antofagasta - Chile

\title{
GAIN OF REGULARITY FOR AN NONLINEAR DISPERSIVE EQUATION KORTEWEG - DE VRIES - BURGERS TYPE *
}

\author{
OCTAVIO PAULO VERA VILLAGRÁN \\ Universidad Católica de la Santísima Concepción - Chile
}

\begin{abstract}
In this papers we study smoothness properties of solutions. We consider the equation of Korteweg - de Vries - Burgers type

(1) $\left\{\begin{array}{l}u_{t}+\partial_{x} f(u)=\epsilon \partial_{x}^{2} u-\delta \partial_{x}^{3} u \\ u(x, 0)=\varphi(x)\end{array}\right.$

with $-\infty<x<+\infty$ and $t>0$. The flux $f=f(u)$ is a given smooth function satisfying certain assumptions to be listed shortly. It is shown under certain additional conditions on $f$ that $C^{\infty}$ - solutions $u(x, t)$ are obtained for all $t>0$ if the initial data $u(x, 0)=\varphi(x)$ decays faster than polinomially on $\mathrm{IR}^{+}=\{x \in \mathrm{IR} ; x>0\}$ and has certain initial Sobolev regularity.
\end{abstract}

Keywords and Phrases : Evolution equations, Lions - Aubin Theorem, Weighted Sobolev Space.

${ }^{*}$ This research was partially supported by DIN 11/2000 Universidad Católica de la Santísima Concepción. Concepción. (Chile). 


\section{Introduction}

In 1976, J. C. Saut and R. Temam [22] have remarked that a solution $u$ of an equation of Korteweg-de Vries type cannot gain or lose regularity: They show that if $u(x, 0)=\varphi(x) \in H^{s}(\mathrm{IR})$ for $s \geq 2$, then $u(\cdot, t) \in H^{s}(\mathrm{IR})$ for all $t>0$. The same results were obtained independently by J. Bona and R. Scott [2] by different methods. For the Korteweg - de Vries ( KdV) equation on the line, T. Kato [16], motivated by work of A. Cohen [6], showed that if $u(x, 0)=\varphi(x) \in L_{b}^{2} \equiv H^{2}(\mathrm{IR}) \cap L^{2}\left(e^{b x} d x\right)(b>0)$ then the solution $u(x, t)$ of the $\mathrm{KdV}$ equation becomes $C^{\infty}$ for all $t>0$. A main ingredient in the proof was the fact that formally the semi-group $S(t)=e^{-t \partial_{x}^{3}}$ in $L_{b}^{2}$ is equivalent to $S_{b}(t)=e^{-t\left(\partial_{x}-b\right)^{3}}$ in $L^{2}$ when $t>0$. One would be inclined to believe this was a special property of the KdV equation. This is not, however, the case. The effect is due to the dispersive nature of the linear part of the equation. S. N. Kruzkov and A. V. Faminskii [20] for $u(x, 0)=\varphi(x) \in L^{2}$ such that $x^{\alpha} \varphi(x) \in L^{2}((0,+\infty))$ is was proven that the weak solution of the $\mathrm{KdV}$ equation constructed there has $l$-continuous space derivatives for all $t>0$ if $l<2 \alpha$. The proof of this result is based on the asymptotic behavior of the Airy function and its derivatives, and on the smoothing effect of the KdV equation found in $[16,20]$. Corresponding work for some special nonlinear Schrödinger equations was done by Hayashi et al. [12,13] and G. Ponce [21]. While the proof of T. Kato appears to depend on special a priori estimates, some of its mystery has been resolved by results of local gain of finite regularity for various others linear and nonlinear dispersive equations due to P. Constantin and J. C. Saut [10], P. Sjolin [23], J. Ginibre and G. Velo [11] and others. However, all of them require growth conditions on the nonlinear term.

All the physically significant dispersive equations and systems known to us have linear parts displaying this local smoothing property. To mention only a few, the KdV, Benjamin-Ono, intermediate long wave, various Boussinesq, and Schrödinger equations are included. Continuing with the idea of W. Craig, T. Kappeler and W. Strauss [9] we study a equation of Korteweg - de Vries - Burgers Type

$$
u_{t}+\partial_{x} f(u)=\epsilon \partial_{x}^{2} u-\delta \partial_{x}^{3} u
$$


with $-\infty<x<+\infty$ and $t>0$. The flux $f=f(u)$ is a given smooth function satisfying certain assumptions to be listed shortly. It is shown under certain additional condition on $f$ that $C^{\infty}$ - solutions $u(x, t)$ are obtained for all $t>0$ if the initial data $u(x, 0)$ decays faster than polinomially on $\mathrm{IR}^{+}=\{x \in \mathrm{IR} ; x>0\}$ and has certain initial Sobolev regularity. In section three we prove an important a priori estimate. In section four we prove basic local-in-time existence and uniqueness results for (1) used in the gain of regularity result in section 7. Specifically, we show that for initial $\varphi(x) \in H^{N}(\mathrm{IR})$, for $N \geq 3$, there exists a unique $u \in L^{\infty}\left([0, T] ; H^{N}(\mathrm{IR})\right)$ where the time of existence depends of the norm of $\varphi(x) \in H^{3}(\mathrm{IR})$. In section five we developed a serie of estimates for solutions of equation (1) in weighted Sobolev norms. We show that solution $u$ in (1) also satisfies a persistence property. Indeed, we prove that if the initial data $\varphi$ lies in a certain weighted Sobolev space, then the unique solution $u$ of the nonlinear equation (1) lies in the same Sobolev space. At the conclusion of sections, we give a formal proof of our gain in regularity theorem for nonlinear equation (1). In section six we state our main results on the gain of regularity for the nonlinear equation (1) and prove the $a$ priori estimate used in the main Theorem 7.2. In the section seven, we state and prove our main results concerning the gain of regularity for solutions to the nonlinear equation (1), including the main estimates for the remainder terms. Specifically, we prove the following principal theorem.

Theorem. (Main Theorem ) Let $T>0$ and $u(x, t)$ be a solution of (1.1) in the region $\mathrm{IR} \times[0, T]$ such that

$$
u \in L^{\infty}\left([0, T] ; H^{3}\left(W_{0 L}\right)\right)
$$

for some $L \geq 2$ and all $\sigma>0$. Then

$$
u \in L^{\infty}\left([0, T] ; H^{3+l}\left(W_{\sigma, L-l, l}\right)\right) \bigcap L^{2}\left([0, T] ; H^{4+l}\left(W_{\sigma, L-l-1, l}\right)\right)
$$

for all $0 \leq l \leq L-1$ 


\section{Preliminaries}

We consider equation of Korteweg - de Vries - Burgers type

$$
u_{t}+[f(u)]_{x}=\epsilon u_{x x}-\delta u_{x x x}
$$

with $-\infty<x<+\infty$ and $t>0$ is an arbitrary positive time, $\epsilon, \delta>0$. The flux $f=f(u) \equiv f(u(x, t))$ is given smooth function satisfying certain assumptions.

Notation 1. We write $\partial=\partial / \partial x ; \quad \partial_{t} u=\partial u / \partial t=u_{t}$

Abreviation 1. We abbreviate $u_{j}=\partial^{j} u=\partial^{j} u / \partial x^{j} \quad ; \quad \partial_{j}=$ $\partial / \partial u_{j}$

Example. If $\partial u / \partial x=u_{1}$ then

$$
\frac{\partial}{\partial x}\left[f\left(u_{0}\right)\right]=\frac{\partial}{\partial x}[f(u)]=\frac{\partial}{\partial u}[f(u)] \frac{\partial}{\partial x}[u]=\frac{\partial}{\partial u}[f(u)] u_{1}=\left(\partial_{0} f\right) u_{1}
$$

Let $T>0$, The assumptions on $f$ are as follow:

A.1 $f: \mathbb{I}^{2} \times[0, T] \mapsto \mathrm{IR}$ is $C^{\infty}$ in all its variables.

A.2 All the derivatives of $f(u, x, t)$ are bounded for $x \in \mathbb{I R}, t \in$ $[0, T]$ and $u$ in a bounded set.

A.3 $x^{N} \partial_{x}^{j} f(0, x, t)$ is bounded for all $N \geq 0, j \geq 0$ and $x \in$ $\mathrm{IR}, t \in(0, T]$.

Lemma 1. These assumptions imply that $f$ has the form

$$
f=u_{0} f_{0}+h \equiv u f_{0}+h
$$

where $f_{0}=f_{0}\left(u_{0}, x, t\right) \equiv f_{0}(u, x, t)$ and $h=h(x, t) . \quad f_{0}$ and $h$ are $C^{\infty}$ and each of their derivatives is bounded for $u$ bounded, $x \in \mathrm{IR}$ and $t \in[0, T]$. 
Proof. Indeed

$$
f_{0}= \begin{cases}\frac{f\left(y_{0}, x, t\right)-f(0, x, t)}{y_{0}} & \text { for } y_{0} \neq 0 \\ \partial_{0} f(0, x, t) & \text { for } y_{0}=0\end{cases}
$$

and $h(x, t)=f(0, x, t)$.

Definition 2.1. An evolution equation enjoys a gain of regularity if its solutions are smoother for all $t>0$ than its initial data.

Definition 2.2. A function $\xi(x, t)$ belong to the weight class $W_{\sigma i k}$ if it is a positive $C^{\infty}$ function on $\mathrm{IR} \times[0, T], \xi_{x} \geq 0$ and there exist constant $c_{j}, 0 \leq j \leq 5$ such that

$(2.4)\left(t\left|\xi_{t}\right|+\left|\partial^{j} \xi\right|\right) / \xi \leq c_{5} \quad$ for $(x, t) \in \mathbf{R} \times[0, T], \forall j \in \mathbf{Z}^{+}$

Remark 1. We shall always take $\sigma \geq 0, k \geq 0$ and $i \geq 1$.

Example 1. Let

$$
\xi(x)= \begin{cases}1+e^{-1 / x} & \text { for } x>0 \\ 1 & \text { for } x \leq 0\end{cases}
$$

then $\xi \in W_{0 i 0}$. 


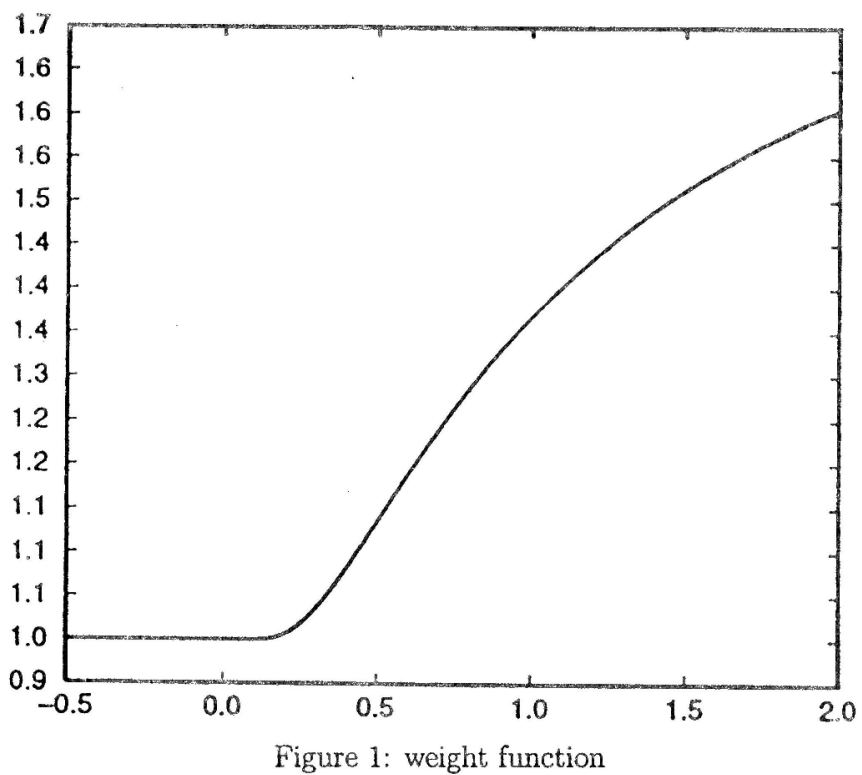

Definition 2.3. Fixed $\xi \in W_{\sigma i k}$ define the space ( $s$ is an integer positive )

$$
\begin{aligned}
& H^{s}\left(W_{\sigma i k}\right)=\left\{v: \mathbb{R} \rightarrow \mathbf{R} ;\|v\|^{2}=\sum_{j=0}^{s} \int_{-\infty}^{+\infty}\left|\partial^{j} v(x)\right|^{2} \xi(x, \cdot) d x<\right. \\
& +\infty\}
\end{aligned}
$$

Remark 2. $H^{s}\left(W_{\sigma i k}\right)$ depends $t$ ( because $\xi=\xi(x, t)$ ).

Lemma 2. For $\xi \in W_{\sigma i 0}$ and $\sigma \geq 0, i \geq 0$ there exists a constant $c$ such that, for $u \in H^{1}\left(W_{\sigma i 0}\right)$

$$
\sup _{x \in \mathbb{R}}\left|\xi u^{2}\right| \leq c \int_{-\infty}^{+\infty}\left(|u|^{2}+|\partial u|^{2}\right) \xi d x
$$


Proof. This result has already been proved in [9].

Definition 2.4. Fixed $T>0$ and $\xi \in W_{\sigma i k}$ define the space $L^{2}\left([0, T] ; H^{s}\left(W_{\sigma i k}\right)\right)=\left\{v=v(x, t), v(\cdot, t) \in H^{s}\left(W_{\sigma i k}\right)\right.$ such that

$$
\begin{gathered}
\left.\|v\|\left\|^{2}=\int_{0}^{T}\right\| v(\cdot, t) \|^{2} d t<+\infty\right\} \\
L^{\infty}\left([0, T] ; H^{s}\left(W_{\sigma i k}\right)\right)=\left\{v=v(x, t), v(\cdot, t) \in H^{s}\left(W_{\sigma i k}\right)\right. \text { such }
\end{gathered}
$$
that

$$
\left.\|v\|_{\infty}=\sup _{t \in[0, T]} \text { ess }\|v(\cdot, t)\|<+\infty\right\}
$$

Remark 3. The usual Sobolev space is $H^{s}(\mathrm{IR})=H^{s}\left(W_{0} 0_{0}\right)$ without a weight.

From now on we consider the following equation

$$
u_{t}+\delta u_{3}-\epsilon u_{2}+\partial_{0} f u_{1}=0
$$

The equation is considered for $-\infty<x<+\infty, t \in[0, T]$ and $T$ is an arbitrary positive time.

\section{An Important a Priori Estimate}

In this section we show an fundamental a priori estimate to demonstrate basic local-in-time existence theorem. Differentiating the equation (2.5) two times leads to

$\left(3.1 \not \partial_{t} u_{2}+\delta u_{5}-\epsilon u_{4}+\partial_{0} f(u) u_{3}+3 \partial_{0}^{2} f(u) u_{1} u_{2}+\partial_{0}^{3} f(u) u_{1}^{3}=0\right.$

Let $u=\wedge v$ where $\wedge=\left(I-\partial^{2}\right)^{-1}$. Then $\partial_{t} u_{2}=-v_{t}+u_{t}$ replacing in (3.1) we have

$$
\begin{aligned}
v_{t}+\delta \wedge v_{5}-\epsilon \wedge v_{4}+\partial_{0} f \wedge & v_{3}+3 \partial_{0}^{2} f \wedge v_{1} \wedge v_{2}+\partial_{0}^{3} f\left(\wedge v_{1}\right)^{3} \\
& -\delta \wedge v_{3}+\epsilon \wedge v_{2}-\partial_{0} f \wedge v_{1}=0
\end{aligned}
$$

The equation (3.2) is linearized by substituting a new variable $w$ in each coefficient

$$
\begin{aligned}
& -v_{t}+\delta \wedge v_{5}-\epsilon \wedge v_{4}+\partial_{0} f(\wedge w) \wedge v_{3}+3 \partial\left(\partial_{0} f(\wedge w)\right) \wedge v_{2} \\
& +\partial^{2}\left(\partial_{0} f(\wedge w)\right) \wedge v_{1}-\delta \wedge v_{3}+\epsilon \wedge v_{2}-\partial_{0} f(\wedge w) \wedge v_{1}=0
\end{aligned}
$$


This linear (3.3) equation which is to be solved at each iteration has the form

$$
\partial_{t} v=\delta \wedge v_{5}-\epsilon \wedge v_{4}+b^{(1)} \wedge v_{3}+b^{(0)}
$$

We consider the following lemma that to help us to set up the iteration scheme.

Lemma 3.1. Given initial data in $\varphi \in H^{\infty}(\mathrm{IR})=\bigcap_{N>0} H^{N}(\mathrm{IR})$ there exists a unique solution of (3.5). Where $b^{(1)}=b^{(1)}(\wedge w)$ and $b^{(0)}=b^{(0)}\left(\wedge w_{2}, \wedge w_{1}, \wedge w\right)$ are smooth bounded coefficients with $w \in$ $H^{\infty}(\mathrm{IR})$. The solution is defined in any time interval in which the coefficients are defined.

The next step is to estimate the corresponding solutions $v=$ $v(x, t)$ of equation (3.3) via the coefficients of that equation.

Lemma 3.2. Let $v, w \in C^{k}\left([0,+\infty) ; H^{N}(\mathrm{IR})\right)$ for all $k, N$ which satisfy (3.3). Let $0<c_{1} \leq \xi \leq c_{2}$. For each integer $\alpha$ there exist positive nondecreasing functions $\mathrm{G}, \mathrm{E}$ and $\mathrm{F}$ such that for all $t \geq 0$

$$
\partial_{t} \int_{R} \xi v_{\alpha}^{2} d x \leq G\left(\|w\|_{\lambda}\right)\|v\|_{\alpha}^{2}+E\left(\|w\|_{\lambda}\right)\|w\|_{\alpha}^{2}+F\left(\|w\|_{\alpha}\right)
$$

where $\|\cdot\|_{\alpha}$ is the norm in $H^{\alpha}(\mathrm{IR})$ and $\lambda=\max \{1, \alpha\}$.

\section{Uniqueness and Local Existence Theorem}

In this section, we study uniqueness and local existence of strong solutions for the problem (2.5). Specifically, we show that for initial $\varphi(x) \in H^{N}(\mathrm{IR})$, for $N \geq 3$, there exists a unique $u \in$ $L^{\infty}\left([0, T] ; H^{N}(\mathrm{IR})\right)$ where the time of existence depends of the norm of $\varphi(x) \in H^{3}(\mathrm{IR})$. These results are used in the proof of Theorem 7.2. First we address the question of uniqueness. 
Theorem 4.1. (Uniqueness) Let $\varphi \in H^{N}(\mathrm{IR})$ with $N \geq 3$ and $0<T<+\infty$. Assume $f$ satisfies A.1-A.3. Then there is at most one strong solution $u \in L^{\infty}\left([0, T] ; H^{N}(\mathrm{IR})\right)$ of $(2.5)$ with initial data $u(x, 0)=\varphi(x)$.

Proof. Assume $u, v \in L^{\infty}\left([0, T] ; H^{N}(\mathrm{IR})\right)$ are two solutions of (2.5) with $u_{t}, v_{t} \in L^{\infty}\left([0, T] ; H^{N-3}(\mathrm{IR})\right)$ and with the same initial data. Then

$$
(u-v)_{t}+\delta(u-v)_{3}-\epsilon(u-v)_{2}+[f(u)-f(v)]_{1}=0
$$

with $(u-v)(x, 0)=0$. By the mean value theorem we have

$$
f(u)-f(v)=\partial f(\theta u+(1-\theta) v)(u-v) \quad 0<\theta<1 .
$$

then there is smooth function $d$ depending smoothly on $u, x, t$ and $v, x, t$ such that (4.1) takes the form

$(4.2 \gamma u-v)_{t}+\delta(u-v)_{3}-\epsilon(u-v)_{2}+d_{1}(u-v)+d(u-v)_{1}=0$

Multiplying (4.2) by $2 \xi(u-v)$ and integrating in $x$ over IR

$$
\begin{array}{r}
2 \int_{R} \xi(u-v)(u-v)_{t} d x+2 \delta \int_{R} \xi(u-v)(u-v)_{3} d x \\
-2 \epsilon \int_{R} \xi(u-v)(u-v)_{2} d x \\
+2 \int_{R} \xi d_{1}(u-v)^{2} d x+2 \int_{R} \xi d(u-v)(u-v)_{1} d x=0
\end{array}
$$

In the firs term we have

$2 \int_{R} \xi(u-v)(u-v)_{t} d x=\partial_{t} \int_{R} \xi(u-v)^{2} d x-\int_{R} \xi_{t}(u-v)^{2} d x$

In the second term integrating by parts

$2 \delta \int_{R} \xi(u-v)(u-v)_{3} d x=$

$=-2 \delta \int_{R} \partial \xi(u-v)(u-v)_{2} d x-2 \delta \int_{R} \xi(u-v)_{1}(u-v)_{2} d x$

$=2 \delta \int_{R} \partial^{2} \xi(u-v)(u-v)_{1} d x+2 \delta \int_{R} \partial \xi(u-v)_{1}^{2} d x+\delta \int_{R} \partial \xi(u-$ $v)_{1}^{2} d x$ 
$=-\delta \int_{R} \partial^{3} \xi(u-v)^{2} d x+3 \delta \int_{R} \partial \xi(u-v)_{1}^{2} d x$

The others terms are treated the similar form. Replacing over (4.3) we have

$$
\begin{aligned}
& \partial_{t} \int_{R} \xi(u-v)^{2} d x-\int_{R} \xi_{t}(u-v)^{2} d x+3 \delta \int_{R} \partial \xi(u-v)_{1}^{2} d x \\
& -\delta \int_{R} \partial^{3} \xi(u-v)^{2} d x+2 \epsilon \int_{R} \xi(u-v)_{1}^{2} d x-\epsilon \int_{R} \partial^{2} \xi(u-v)^{2} d x \\
& +2 \int_{R} \xi d_{1}(u-v)^{2} d x-\int_{R} \partial(\xi d)(u-v)^{2} d x=0
\end{aligned}
$$
then

$$
\begin{array}{r}
\partial_{t} \int_{R} \xi(u-v)^{2} d x+\int_{R}(3 \delta \partial \xi+2 \epsilon \xi)(u-v)_{1}^{2} d x=\int_{R}\left[\xi_{t}+\delta \partial^{3} \xi\right. \\
\left.+\epsilon \partial^{2} \xi-2 \xi d_{1}+\partial(\xi d)\right](u-v)^{2} d x
\end{array}
$$

Using the assumptions on $f$ and for a suitably chosen constant $c$, we have

$\partial_{t} \int_{\mathbb{I}} \xi(u-v)^{2} d x+\int_{\mathbb{I}}(3 \delta \partial \xi+2 \epsilon \xi)(u-v)_{1}^{2} d x \leq c \int_{\mathbb{R}} \xi(u-v)^{2} d x$

By Gronwall's inequality and the fact $(u-v)$ vanishes at $t=0$ it follows that $u=v$. This proves uniqueness.

To stablish the local existence of strong solutions to (2.5) we use of the a priori estimate together with a approximation procedure.

We construct the mapping $T: L^{\infty}\left([0, T] ; H^{s}(\mathrm{IR})\right) \rightarrow L^{\infty}([0, T]$ ; $\left.H^{s}(\mathrm{IR})\right)$ by defining that

$$
\begin{aligned}
& u^{(0)}=\varphi(x) \\
& u^{(n)}=T\left(u^{(n-1)}\right) \quad n \geq 1
\end{aligned}
$$

where $u^{(n-1)}$ is in the position of $w$ in equation (3.3) and $u^{(n)}$ is in the position of $v$ which is the solution of equation (3.3). By to lemma 3.1., $u^{(n)}$ exists and is unique in $C\left((0,+\infty) ; H^{N}(\mathrm{IR})\right)$. A choice of $c_{0}$ and the use of the a priori estimate in $\S 3$ show that $T: \mathrm{IB}_{c_{0}}(0) \rightarrow \mathrm{IB}_{c_{0}}(0)$ with $\mathrm{IB}_{c_{0}}(0)$ a bounded ball in $L^{\infty}\left([0, T] ; H^{s}(\mathrm{IR})\right)$.

We know state our existence theorem for equation (2.5).

Theorem 4.2. (Local Existence) Assume $f$ satisfies A.1 - A.4. Let $\mathrm{N}$ be an integers $\geq 3$. If $\varphi \in H^{N}(\mathrm{IR})$, then there is $T>0$ and $u$ such that $u$ is a strong solution of (2.5). $u \in L^{\infty}\left([0, T] ; H^{N}(\mathrm{IR})\right)$ with initial data $u(x, 0)=\varphi(x)$.

Proof. We prove that for $\varphi \in H^{\infty}(\mathrm{IR})=\bigcap_{k \geq 0} H^{k}(\mathrm{IR})$ there exists a solution 
$u \in L^{\infty}\left([0, T] ; H^{N}(\mathrm{IR})\right)$ with initial data $u(x, 0)=\varphi(x)$ and which a time of existence $T>0$ which only depends on the norm of $\varphi$.

We define a sequence of approximations to equation (3.3) as

$$
\begin{array}{r}
-v_{t}^{(n)}+\delta \wedge v_{5}^{(n)}-\epsilon \wedge v_{4}^{(n)}+\partial_{0} f\left(\wedge v^{(n-1)}\right) \wedge v_{3}^{(n)}+3 \partial\left(\partial_{0} f\left(\wedge v^{(n-1)}\right)\right) \\
\wedge v_{2}^{(n)}+\partial^{2}\left(\partial_{0} f\left(\wedge v^{(n-1)}\right)\right) \wedge v_{1}^{(n)}-\delta \wedge v_{3}^{(n)}+\epsilon \wedge v_{2}^{(n)}-\partial_{0} f\left(\wedge v^{(n-1)}\right) \\
\wedge v_{1}^{(n)}=0
\end{array}
$$

with initial data $v^{(n)}(x, 0)=\varphi(x)-\partial^{2} \varphi(x)$.

The first approximation is given by $v^{(0)}(x, 0)=\varphi(x)-\partial^{2} \varphi(x)$.

Equation (4.4) is a linear equation at each iteration which can be solved in any interval of time in which the coefficients are defined. This is shown in lemma 3.1. By lemma 3.2 follows that

$$
\begin{array}{r}
\partial_{t} \int_{R} \xi\left[v_{\alpha}^{(n)}\right]^{2} d x \leq G\left(\left\|v^{(n-1)}\right\|_{\lambda}\right)\left\|v^{(n)}\right\|_{\alpha}^{2}+E\left(\left\|v^{(n-1)}\right\| \|_{\lambda}\right)\left\|v^{(n-1)}\right\|_{\alpha}^{2} \\
+F\left(\left\|v^{(n-1)}\right\|_{\alpha}\right)
\end{array}
$$

Choose $\alpha=1$ and let $c_{0} \geq\left\|\varphi-\partial^{2} \varphi\right\|_{1} \geq\|\varphi\|_{3}$. For each iterate $n,\left\|v^{(n)}(\cdot, t)\right\|$ is continuous in $t \in[0, T]$ and $\left\|v^{(n)}(\cdot, 0)\right\| \leq c_{0}$. Define $c_{3}=\frac{c_{2}}{2 c_{1}} c_{0}^{2}+1$. Let $T^{(n)}$ be the maximum time such that $\left\|v^{(k)}(\cdot, t)\right\|_{1} \leq c_{3}$ for $0 \leq t \leq T^{(n)}, 0 \leq k \leq n$. Integrating (4.5) over $[0, t]$ we have for $0 \leq t \leq T^{(n)}$ and $j=0,1, \ldots$ follows that

$$
\begin{gathered}
\int_{R} \xi(x, t)\left[v_{j}^{(n)}(x, t)\right]^{2} d x \leq \int_{R} \xi(x, 0)\left[v_{j}^{(n)}(x, 0)\right]^{2} d x+ \\
\int_{0}^{t} G\left(\left\|v^{(n-1)}\right\|_{1}\right)\left\|v^{(n)}\right\|_{j}^{2} d s+\int_{0}^{t} E\left(\left\|v^{(n-1)}\right\|_{1}\right)\left\|v^{(n-1)}\right\|_{j}^{2} d s+ \\
\int_{0}^{t} F\left(\left\|v^{(n-1)}\right\|_{j}\right) d s
\end{gathered}
$$

hence

$$
\begin{gathered}
c_{1} \int_{R}\left[v_{j}^{(n)}\right]^{2} d x \leq \int_{R} \xi\left[v_{j}^{(n)}\right]^{2} d x \leq \int_{R} \xi(x, 0)\left[v_{j}^{(n)}(x, 0)\right]^{2} d x+ \\
\int_{0}^{t} G\left(\left\|v^{(n-1)}\right\|_{1}\right)\left\|v^{(n)}\right\|_{j}^{2} d s+\int_{0}^{t} E\left(\left\|v^{(n-1)}\right\|_{1}\right)\left\|v^{(n-1)}\right\|_{j}^{2} d s+ \\
\int_{0}^{t} F\left(\left\|v^{(n-1)}\right\|_{j}\right) d s
\end{gathered}
$$

this way

$\int_{\mathbb{R}}\left[v_{j}^{(n)}\right]^{2} d x \leq \frac{c_{2}}{c_{1}} \int_{\mathbb{R}}\left[v_{j}^{(n)}(x, 0)\right]^{2} d x+\frac{G\left(c_{3}\right)}{c_{1}} c_{3}^{2} t+\frac{E\left(c_{3}\right)}{c_{1}} c_{3}^{2} t+\frac{F\left(c_{3}\right)}{c_{1}} t$ 
and we obtain for $j=0,1$.

$$
\left\|v^{(n)}\right\|_{1} \leq \frac{c_{2}}{c_{1}} c_{0}^{2}+2 \frac{G\left(c_{3}\right)}{c_{1}} c_{3}^{2} t+2 \frac{E\left(c_{3}\right)}{c_{1}} c_{3}^{2} t+2 \frac{F\left(c_{3}\right)}{c_{1}} t
$$

Choosing $T=T\left(c_{0}\right)$ sufficiently small, but $T$ not depending on $n$, one concludes that

$$
\left\|v^{(n)}\right\|_{1} \leq c
$$

for $0 \leq t \leq T$. This shows that $T^{(n)} \geq T$. We have shown that $v^{(n)}$ is a bounded sequence in $L^{\infty}\left([0, T] ; H^{1}(\mathrm{IR})\right)$. Hence of (4.6) there is a weak ${ }^{*}$ convergent subsequence $v^{\left(n_{j}\right)} \stackrel{\text { def }}{=} v^{(n)}$ such that $v^{(n)} \stackrel{*}{\rightarrow}$ $v \quad$ weak in $L^{\infty}\left([0, T] ; H^{1}(\mathrm{IR})\right)$.

Claim. $u=\wedge v$ is the solution we are looking for.

Proof. We first need to show that $v$ is a solution of (3.2). We do so by showing that each term in (4.4) converges to its corret limit. By equation (4.4), $\partial_{t} v^{(n)}$ is a sum of terms each of which is the product of a coefficient, bounded uniformly in $n$, so that the sequence $\partial_{t} v^{(n)}$ is bounded in $L^{2}\left([0, T] ; H^{-2}(\mathrm{IR})\right)$. By LionsAubin's compactness theorem, there is a subsequence $v^{\left(n_{j}\right)} \stackrel{\text { def }}{=} v^{(n)}$ such that $v^{(n)} \longrightarrow v$ strongly in $L^{2}\left([0, T] ; H_{l o c}^{1 / 2}(\mathrm{IR})\right)$. Therefore, for a subsequence, $v^{\left(n_{j}\right)} \stackrel{\text { def }}{=} v^{(n)}$ we have $v^{(n)} \longrightarrow v$ a. e. in $x$ and $t$. It follows that the fourth term on the right hand side of (4.4) $\partial_{0} f\left(\wedge v^{(n-1)}\right) \wedge v_{3}^{(n)} \rightarrow \partial_{0} f(\wedge v) \wedge v_{3}$ weak in $L^{2}\left([0, T] ; L_{l o c}^{1}(\mathrm{IR})\right)$ as $\wedge v_{3}^{(n)} \rightarrow \wedge v_{3}$ weak in $L^{2}\left([0, T] ; H^{-2}(\mathrm{IR})\right)$ and $\partial_{0} f\left(\wedge v^{(n-1)}\right) \longrightarrow$ $\partial_{0} f(\wedge v)$ strong in $L^{2}\left([0, T] ; H_{l o c}^{2}(\mathrm{IR})\right)$. Similarly all other terms in (4.4) converge to their corrects limits, implying $v_{t}^{(n)} \rightarrow v_{t}$ weak in $L^{2}\left([0, T] ; L_{l o c}^{1}(\mathrm{IR})\right)$. This way we have $(2.5)$ for $u=\wedge v$.

We prove that there exists a solution of the equation (2.5) with $u \in L^{\infty}\left([0, T] ; H^{N}(\mathrm{IR})\right)$ and $N \geq 4$, where $T$ depends only on the norm of $\varphi$. We already know that there is a solution ( previously ) $u \in L^{\infty}\left([0, T] ; H^{3}(\mathrm{IR})\right)$. It is suffices to prove that the approximating sequence $v^{(n)}$ is bounded in $L^{\infty}\left([0, T] ; H^{N-2}(\mathrm{IR})\right)$. Take $\alpha=N-2$ and consider (4.5) for $\alpha \geq 2$. By the same arguments as for $\alpha=1$ we conclude that there exists $T^{(\alpha)}>0$ depending on the norm of $\varphi$ 
but independent $n$ such that $\left\|v^{(n)}\right\|_{\alpha} \leq c$ for all $0 \leq t \leq T^{(\alpha)}$. Thus $v \in L^{\infty}\left(\left[0, T^{(\alpha)}\right] ; H^{\alpha}(\mathrm{IR})\right)$. Now denote by $0 \leq T^{*(\alpha)} \leq+\infty$ the maximal number such that for all $0<T \leq T^{*(\alpha)}$

$$
u=\wedge v \in L^{\infty}\left([0, T] ; H^{N}(\mathrm{IR})\right)
$$

We claim that $T^{(1)} \leq T^{*(\alpha)}$ for all $\alpha \geq 2$. Thus $T$ can be chosen depending only on norm of $\varphi$. Approximating $\varphi$ by $\left\{\varphi_{j}\right\} \in$ $C_{0}^{\infty}(\mathrm{IR})$ such that $\left\|\varphi-\varphi_{j}\right\|_{H^{N}(\mathrm{IR})} \stackrel{j \longrightarrow+\infty}{\longrightarrow} 0$. Let $u_{j}$ be a solution of $(2.5)$ with $u_{j}(x, 0)=\varphi_{j}(x)$. According to the above argument, there exists $T$ which is independent of $n$ but depending only $\sup _{j}\left\|\varphi_{j}\right\|$ such that $u_{j}$ exists on $[0, T]$ and a subsequence $u_{j} \stackrel{j \longrightarrow+\infty}{\longrightarrow} u$ in $L^{\infty}\left([0, T] ; H^{N}(\mathrm{IR})\right)$. As a consequence of Theorem 4.1 and Theorem 4.2 and its proof one gets.

Corollary 4.3. Let $\varphi \in H^{3}(\mathrm{IR}) \cap H^{N}(\mathrm{IR})$ with $N \geq 3$ such that $\varphi^{(\gamma)} \longrightarrow \varphi$ in $H^{N}(\mathrm{IR})$. Let $u$ and $u^{(\gamma)}$ be the corresponding unique solutions given by Theorems 4.1 and 4.2 in $L^{\infty}\left([0, T] ; H^{N}(\mathrm{IR})\right)$ with $T$ depending only on $\sup _{\gamma}\left\|\varphi^{(\gamma)}\right\|_{H^{3}(\mathrm{IR})}$ then

$$
u^{(\gamma)} \stackrel{*}{\rightarrow} u \quad \text { weak in } L^{\infty}\left([0, T] ; H^{N}(\mathrm{IR})\right)
$$

and

$$
u^{(\gamma)} \longrightarrow u \quad \text { strong in } L^{2}\left([0, T] ; H^{N+1}(\mathrm{IR})\right)
$$

\section{Main Inequality}

Lemma 5.1. Let $u$ be a solution of the initial value problem (2.5). Then we have the following inequality.

$$
\partial_{t} \int_{\mathbb{I} \mathbf{R}} \xi u_{\alpha}^{2} d x+\int_{\mathbb{I}} \eta u_{\alpha+1}^{2} d x+\int_{\mathbb{I}} \theta u_{\alpha}^{2} d x+\int_{\mathbb{R}} R d x \leq 0
$$

with

$$
\begin{aligned}
& \eta=\left(3 \delta+2 \frac{\epsilon}{c}\right) \partial \xi \\
& \theta=-\xi_{t}-\delta \partial^{3} \xi-\epsilon \partial^{2} \xi-\partial\left(\xi \partial_{0} f\right) \\
& R=O\left(u_{\alpha}, \ldots\right)
\end{aligned}
$$


Proof. Taking $\alpha$-derivatives of the equation (2.5) (for $\alpha \geq 3$ ) over $x \in \mathbb{I R}$

$$
\partial_{t} u_{\alpha}+\delta u_{\alpha+3}-\epsilon u_{\alpha+2}+\partial_{0} f u_{\alpha+1}+O\left(u_{\alpha}, u_{\alpha-1}, \ldots\right)=0
$$

Multiply (5.1) by $2 \xi u_{\alpha}$, integrate over $x \in \mathbb{I R}$ we have

$$
\begin{gathered}
2 \int_{\mathbb{R}} \xi u_{\alpha} \partial_{t} u_{\alpha} d x+2 \delta \int_{\mathbb{I R}} \xi u_{\alpha} u_{\alpha+3} d x-2 \epsilon \int_{\mathbb{R}} \xi u_{\alpha} u_{\alpha+2} d x+ \\
2 \int_{\mathbb{I}} \xi u_{\alpha} \partial_{0} f u_{\alpha+1} d x+\int_{\mathbb{R}} R d x=0
\end{gathered}
$$

integrating by parts we have

$\partial_{t} \int_{\mathbb{R}} \xi u_{\alpha}^{2} d x+\int_{\mathbb{R}}(3 \delta \partial \xi+2 \epsilon \xi) u_{\alpha+1}^{2} d x+\int_{\mathbb{R}} \theta u_{\alpha}^{2} d x+\int_{\mathbb{R}} R d x=0$

with $\theta=-\xi_{t}-\delta \partial^{3} \xi-\epsilon \partial^{2} \xi-\partial\left(\xi \partial_{0} f\right)$. Using (2.4) follows

$\partial_{t} \int_{\mathbb{R}} \xi u_{\alpha}^{2} d x+\int_{\mathbb{R}}\left(3 \delta+2 \frac{\epsilon}{c}\right) \partial \xi u_{\alpha+1}^{2} d x+\int_{\mathbb{R}} \theta u_{\alpha}^{2} d x+\int_{\mathbb{R}} R d x \leq 0$

where we obtain main inequality.

$$
\partial_{t} \int_{R} \xi u_{\alpha}^{2} d x+\int_{R} \eta u_{\alpha+1}^{2} d x+\int_{R} \theta u_{\alpha}^{2} d x+\int_{R} R d x \leq 0
$$

with

$$
\begin{aligned}
& \eta=\left(3 \delta+2 \frac{\epsilon}{c}\right) \partial \xi \\
& \theta=-\xi_{t}-\delta \partial^{3} \xi-\epsilon \partial^{2} \xi-\partial\left(\xi \partial_{0} f\right) \\
& R=O\left(u_{\alpha}, \ldots\right)
\end{aligned}
$$

Lemma 5.2. If $\eta \in W_{\sigma i k}$ is an arbitrary weight function, then there exist $\xi \in W_{\sigma, i+1, k}$ which satisfies

$$
\eta=\left(3 \delta+2 \frac{\epsilon}{c}\right) \partial \xi
$$

Proof. Indeed

$$
\xi=\frac{1}{\left(3 \delta+2 \frac{\epsilon}{c}\right)} \int_{-\infty}^{x} \eta(y, t) d y
$$


Lemma 5.3. The expression $R$ in the main inequality is a sum of terms of the form

$$
\xi \partial_{0}^{p_{0}} \partial_{x}^{\gamma} f u_{\nu_{1}} u_{\nu_{2}} \ldots u_{\nu_{p-1}} u_{\nu_{p}} u_{\alpha}
$$

where $1 \leq \nu_{1} \leq \nu_{2} \leq \ldots \leq \nu_{p} \leq \alpha$.

$$
\begin{gathered}
p=p_{0} \geq 1, \quad \gamma=\alpha+1 \\
\nu_{1}+\nu_{2}+\ldots+\nu_{p}=\alpha+1 \\
p+\nu_{p-1}+\nu_{p} \leq \alpha+3
\end{gathered}
$$

\section{Persistence Theorem}

As a starting point for the a priori gain of regularity results that will be discussed in next section, we need to develop some estimates for solutions of the equation (2.5) in weighted Sobolev norms. The existence of these weighted estimated is often called the persistence of a property of the initial data $\varphi$. We show that if $\varphi \in H^{3}(\mathrm{IR}) \cap H^{L}\left(W_{0 i 0}\right)$ for $L \geq$ $3, i \geq 1$ then the solution $u(\cdot, t)$ evolves in $H^{L}\left(W_{0 i 0}\right)$ for $t \in[0, T]$. The time interval of such persistence is at least as long as the interval guaranteed by the existence theorem 4.2 .

Theorem 6.1. (Persistence) Let $i \geq 1$ and $L \geq 3$ be nonnegative integers, $0<T<+\infty$. Assume that $u$ is the solution to $(2.5)$ in $L^{\infty}\left([0, T] ; H^{3}(\mathrm{IR})\right)$ with initial data $\varphi(x)=u(x, 0) \in$ $H^{3}(\mathrm{IR})$. If $\varphi(x) \in H^{L}\left(W_{0 i 0}\right)$ then

$$
u \in L^{\infty}\left([0, T] ; H^{3}(R) \bigcap H^{L}\left(W_{0 i 0}\right)\right)
$$

where $\sigma$ is arbitrary, $\eta \in W_{\sigma, i-1,0}$ for $i \geq 1$. 


\section{The Main Theorem}

In this section we state and prove our main theorem, which tells us that if the initial data $u(x, 0)$ decays faster than polinomially on $\mathrm{IR}^{+}=$ $\{x \in \mathrm{IR} ; x>0\}$ and possesses certain initial Sobolev regularity, then the solution $u(x, t) \in C^{\infty}$ for all $t>0$.

In the case of main theorem, we take $4 \leq \alpha \leq L+2$. For $\alpha \leq L+2$, we take any

$$
\eta \in W_{\sigma, L-\alpha+2, \alpha-3} \Longrightarrow \xi \in W_{\sigma, L-\alpha+3, \alpha-3}
$$

Lemma 7.1. (Estimate of Error Terms ) Let $4 \leq \alpha \leq L+2$ and the weight functions are chosen as in (7.1), then

$$
\left|\int_{0}^{T} \int_{\mathbb{I} R}\left(\theta u_{\alpha}^{2}+R\right) d x d t\right| \leq c
$$

where $c$ depends only on the norms of $u$ in

$$
L^{\infty}\left([0, T] ; H^{\beta}\left(W_{\sigma, L-\beta+2, \beta-3}\right)\right) \bigcap L^{2}\left([0, T] ; H^{\beta+1}\left(W_{\sigma, L-\beta+1, \beta-3}\right)\right)
$$

for $3 \leq \beta \leq \alpha-1$, and the norms of $u$ in $L^{\infty}\left([0, T] ; H^{3}\left(W_{0 L_{0}}\right)\right)$.

Theorem 7.2. (Main Theorem) Let $T>0$ and $u(x, t)$ be a solution of $(2.5)$ in the region $\mathrm{IR} \times[0, T]$ such that

$$
u \in L^{\infty}\left([0, T] ; H^{3}\left(W_{0 L}\right)\right)
$$

for some $L \geq 2$ and all $\sigma>0$. Then

$$
u \in L^{\infty}\left([0, T] ; H^{3+l}\left(W_{\sigma, L-l, l}\right)\right) \bigcap L^{2}\left([0, T] ; H^{4+l}\left(W_{\sigma, L-l-1, l}\right)\right)
$$

for all $0 \leq l \leq L-1$

Remark 7.1. If the assumption (7.9) holds for all $L \geq 2$, the solution is infinitely differentiable in the $x$-variable. From the equation (2.5) itself the solution $C^{\infty}$ in both of its variables. 
Proof. (Induction on $\alpha$ )

For $\alpha=4$. Let $u$ be a solution (2.5) satisfying (7.9), the equation itself implies that $u_{t} \in L^{\infty}\left([0, T] ; L^{2}\left(W_{0 L_{0}}\right)\right)$ where $u \in$ $L^{\infty}\left([0, T] ; H^{3}\left(W_{0 L_{0}}\right)\right), \quad u_{t} \in L^{\infty}\left([0, T] ; L^{2}\left(W_{0 L_{0}}\right)\right) \quad$ then $u \in$ $C\left([0, T] ; L^{2}\left(W_{0 L} 0\right)\right) \cap C_{w}\left([0, T] ; H^{3}\left(W_{0 L}\right)\right)$. Hence $u:[0, T] \mapsto$ $H^{3}\left(W_{0} L_{0}\right)$ is a weakly continuous function. In particular $u(\cdot, t) \in$ $H^{3}\left(W_{0} L_{0}\right)$ for all $t$. Let $t_{0} \in(0, T)$ and $u\left(\cdot, t_{0}\right) \in H^{3}\left(W_{0} L_{0}\right)$, then there are $\left\{\varphi^{(n)}\right\} \subset C_{0}^{\infty}(\mathrm{IR})$ such that

$$
\varphi^{(n)}(\cdot) \longrightarrow u\left(\cdot, t_{0}\right) \text { in } H^{3}\left(W_{0 L 0}\right)
$$

Let $u^{(n)}(x, t)$ be a unique solution of $(2.5)$ with $u^{(n)}\left(x, t_{0}\right)=\varphi^{(n)}(x)$. Then by theorem 4.1 , theorem 4.2 to exist in a time interval $\left[t_{0}, t_{0}+\right.$ $\delta$ ] where $\delta>0$ does not depend on $n$ and $u$ be a unique solution of (2.5) $u^{(n)} \in L^{\infty}\left(\left[t_{0}, t_{0}+\delta\right] ; H^{3}\left(W_{0 L}\right)\right)$ with

$$
u^{(n)}\left(x, t_{0}\right) \equiv \varphi^{(n)}(x) \longrightarrow u\left(x, t_{0}\right) \equiv \varphi(x) \quad \text { in } \quad H^{3}\left(W_{0 L}\right)
$$

Now, by theorem 6.1 , we have

$$
u^{(n)} \in L^{\infty}\left(\left[t_{0}, t_{0}+\delta\right] ; H^{3}\left(W_{0 L 0}\right)\right) \bigcap L^{2}\left(\left[t_{0}, t_{0}+\delta\right] ; H^{4}\left(W_{\sigma, L-1,0}\right)\right)
$$

with a bound that depends only on the norm of $\varphi^{(n)}$ in $H^{3}\left(W_{0} L_{0}\right)$. Furthermore, Theorem 6.1 guarantees the non-uniform bounds

$$
\sup _{\left[t_{0}, t_{0}+\delta\right]} \sup _{x}\left(1+\left|x_{+}\right|\right)^{k}\left|\partial^{\alpha} u^{(n)}(x, t)\right|<+\infty
$$

for each $n, k$ and $\alpha$. The main inequality (5.2) and the estimate (7.2) are therefore valid for each $u^{(n)}$ in the interval $\left[t_{0}, t_{0}+\delta\right]$. $\eta$ may be chosen arbitrarily in its weight class (7.1) and then $\xi$ is defined by (5.4) and the constant $c_{1}, c_{2}, c_{3}, c_{4}$ are independent of $n$. From (5.2) and (7.2) we have

$$
\sup _{\left[t_{0}, t_{0}+\delta\right]} \int_{R} \xi\left[u_{\alpha}^{(n)}\right]^{2} d x+\int_{t_{0}}^{t_{0}+\delta} \int_{R} \eta\left[u_{\alpha+1}^{(n)}\right]^{2} d x d t \leq c
$$

where by (7.2) $c$ independ of $n$. This estimate (7.10) is proved by induction for $\alpha=4,5,6, \ldots$ Thus $u^{(n)}$ is also bounded in

$$
\begin{array}{r}
L^{\infty}\left(\left[t_{0}, t_{0}+\delta\right] ; H^{\alpha}\left(W_{\sigma, L-\alpha+3, \alpha-3}\right)\right) \cap L^{2}\left(\left[t_{0}, t_{0}+\delta\right] ;\right. \\
\left.H^{\alpha+1}\left(W_{\sigma, L-\alpha+2, \alpha-3}\right)\right)
\end{array}
$$


for $\alpha \geq 4$. Since

$$
u^{(n)} \longrightarrow u \text { in } L^{\infty}\left(\left[t_{0}, t_{0}+\delta\right] ; H^{3}\left(W_{0 L 0}\right)\right)
$$

By corollary 4.3 it follows that $u$ belong to the space (7.11). Since $\delta$ is fixed, this result is valid over the whole interval $[0, T]$.

Acknowledgments: The author is very grateful to professors Felipe Linares (IMPA) and Mauricio Sepúlveda (U. de Concepción) for their valuable suggestions.

\section{References}

[1] J. Bona, G. Ponce, J. C. Saut and M. M. Tom. A model system for strong interaction between internal solitary waves, Comm. Math. Phys. Appl. Math., 143, pp. 287-313, (1992).

[2] J. Bona and R. Scott. Solutions of the Korteweg - de Vries equation in fractional order Sobolev space, Duke Math. J. 43, 87-99, (1976).

[3] J. Bona and R. Smith. The initial value problem for the Korteweg - de Vries equation, Philos. Trans. Royal Soc. London, Ser. A, 278, pp. 555-601, (1975).

[4] J. Bona and J.C. Saut. Dispersive blow-up of solutions of generalized Korteweg - de Vries equation, Journal of Diff. equations., 103, pp. 3-57, (1993).

[5] H. Cai. Dispersive smoothing effect for generalized and high order KdV type equations. Journal of Diff. equations., 136, pp.191-221, (1997)

[6] A. Cohen. Solutions of the Korteweg - de Vries equations from irregular data, Duke Math., J., Vol. 45, springer, pp. 149-181, (1991).

[7] W. Craig and J. Goodman. Linear dispersive equations of Airy Type, J. Diff. equations., Vol. 87, pp. 38-61, (1990). 
[8] W. Craig, T. Kappeler and W. Strauss. Infinite gain of regularity for dispersive evolution equations, Microlocal Analysis and Nonlinear waves, I.M.A., Vol. 30, springer, pp. 47-50, (1991).

[9] W. Craig, T. Kappeler and W. Strauss. Gain of regularity for equations of Korteweg - de Vries type, Ann. Inst. Henri Poincaré, Vol. 9, Nro. 2, pp. 147-186, (1992).

[10] P. Constantin and J. C. Saut. Local smoothing properties of dispersive equations, Journal A.M.S., Nro. 1, pp. 413-439, (1988).

[11] J. Ginibre and G. Velo. Conmutator expansions and smoothing properties of generalized Benjamin - Ono equations. Ann. Inst. Henri Poincaré, Vol. 51, Nro. 2, pp. 221-229, (1989).

[12] N. Hayashi, K. Nakamitsu and M. Tsutsumi. On solutions on the initial value problem for the nonlinear Schrodinger equations in One Space Dimension, Math. Z., Vol. 192, pp. 637-650, (1986).

[13] N. Hayashi, K. Nakamitsu and M. Tsutsumi. On solutions of the initial value problem for nonlinear Schrodinger equations, J. of Funct. Anal., Vol. 71, pp. 218-245, (1987).

[14] N. Hayashi and T. Ozawa. Smoothing effect for some Schrodinger equations, J. of Funct. Anal., Vol. 85, pp. 307-348, (1989).

[15] T. Kato. Quasilinear equations of evolutions with applications to partial differential equations, Lecture notes in mathematics, Springer-Verlag, Vol. 448, pp. 27-50, (1975).

[16] T. Kato. On the Cauchy problem for the (generalized) Korteweg - de Vries equations, Adv. in Math. Suppl. Studies, Studies in Appl. Math., Vol. 8, pp. 93-128, (1983).

[17] T. Kato and G. Ponce. Commutator estimates and the Euler and Navier-Stokes equations, Comm. Pure Applied Math., Vol. 41, pp. 891-907, (1988).

[18] C. Kenig, G. Ponce and L. Vega. On the (generalized) Korteweg - de Vries equation, Duke Math. J., Vol. 59 (3), pp. 585-610, (1989). 
[19] C. Kenig, G. Ponce and L. Vega. Oscillatory integrals and regularity equations, Indiana Univ. Math., J., Vol. 40, pp. 33-69, (1991)

[20] S. N. Kruzhkov and A.V. Faminskii. Generalized solutions to the Cauchy problem for the Korteweg - de Vries equations, Math. U.S.S.R. Sbornik, Vol. 48, pp. 93-138, (1984).

[21] G. Ponce. Regularity of solutions to nonlinear dispersive equations, J. Diff. Eq., Vol. 78, pp. 122-135, (1989).

[22] J.C. Saut and R. Temam. Remark on the Korteweg - de Vries equation, Israel J. Math., Vol. 24, pp. 78-87, (1976).

[23] P. Sjolin. Regularity of solutions to the Schrodinger equation. Duke Math. J., Vol. 55, pp. 699-715, (1987).

[24] R. Temam. Sur un probleme non-lineaire, J. Math. Pures Appl., Vol. 48, pp. 159-172, (1969).

Received : December 1999.

\section{Octavio Vera Villagrán}

Facultad de Ingeniería

Universidad Católica de la Santísima Concepción

Paicaví 3000

Concepción

Chile

e-mail : overa@david.ucsc.cl 